\title{
Climate of Eccentric Terrestrial Planets with Carbonate-Silicate Geochemical Cycle
}

\author{
Shintaro Kadoya ${ }^{1}$, Eiichi Tajika ${ }^{2}$, and Yoshiyasu Watanabe ${ }^{3}$ \\ ${ }^{1}$ Department of Earth \& Planetary Science, University of Tokyo, Kiban bldg. 408, 5-1-5, \\ Kashiwanoha, Kashiwa, Chiba, 277-8561, Japan \\ email: kadoya@astrobio.k.u-tokyo.ac.jp \\ ${ }^{2}$ Department of Complecity Science \& Engineering, University of Tokyo, Kiban bldg. 409, \\ 5-1-5, Kashiwanoha, Kashiwa, Chiba, 277-8561, Japan \\ email: tajika@k.u-tokyo.ac.jp \\ ${ }^{3}$ Department of Earth \& Planetary Science, University of Tokyo, Kiban bldg. 408, 5-1-5, \\ Kashiwanoha, Kashiwa, Chiba, 277-8561, Japan \\ email: y-watanabe@astrobio.k.u-tokyo.ac.jp
}

\begin{abstract}
Recent discovery of extrasolar planets indicates that some of them have much higher eccentricity than the planets in the solar system. Here, we investigate the climate of such eccentric terrestrial planets with oceans and carbonate-silicate geochemical cycles. We find that the climate of the planets are dependent on the annual mean insolation as shown in previous works. We also find that the planets orbiting slightly further from our Sun than the Earth are globally ice-covered even if the carbonate-silicate geochemical cycle works under the same $\mathrm{CO}_{2}$ degassing rate as on the present Earth. However, when the $\mathrm{CO}_{2}$ degassing rate is higher, the planets avoid being globally ice-covered owing to the high level.
\end{abstract}

Keywords. terrestrial planets - atmosphere; exoplanet atmosphere; habitability

\section{Introduction}

Since 1995, more than 2000 candidates of extrasolar planets have been found. Some of these planetary candidates have high eccentricities, and receive insolation that changes seasonally. Such planets are called eccentric planets, and the characteristic features of their climate have been discussed in papers such as Williams \& Pollard (2002) and Dressing et al. (2010). Williams \& Pollard (2002) showed that the climate of an eccentric terrestrial planet depends mainly on the annual mean insolation, and Dressing et al. (2010) concluded that an eccentric terrestrial planet far from the central star could have water seasonally on its surface. These authors, however, considered the planets with a constant partial pressure of $\mathrm{CO}_{2}$, although the partial pressure of $\mathrm{CO}_{2}$ on Earth-like planets may be controlled by carbonate-silicate geochemical cycle, and may change on long timescale according to the conditions such as insolation, degassing flux of $\mathrm{CO}_{2}$ and so on. In other words, the results may be only tentative and should change owing to change of partial pressure of $\mathrm{CO}_{2}$ through carbonate-silicate geochemical cycle, and the long-term stable climate should be different from their results. In this study, we therefore investigate the climate of such eccentric terrestrial planets with oceans, considering the carbonate-silicate geochemical cycle. 


\section{Model}

We use a one-dimensional energy balance climate model (e.g., North et al. 1981; Williams \& Kasting 1997):

$$
C(\phi, T) \frac{\partial T(\phi, t)}{\partial t}=[1-A(T)] S(t)-I\left(T, \mathrm{pCO}_{2}\right)+\operatorname{div} F(T)
$$

where $T$ is temperature, $\phi$ is latitude, $t$ is time, $C$ is heat capacity, $A$ is albedo, $S$ is insolation, $I$ is planetary radiation, and $F$ is meridional heat transport. The greenhouse gas is assumed here to be $\mathrm{CO}_{2}$ and $\mathrm{H}_{2} \mathrm{O}$, and the amount of $\mathrm{CO}_{2}$ in the atmosphere is controlled by carbonate-silicate geochemical cycle, in which $\mathrm{CO}_{2}$ degassing is given, and $\mathrm{CO}_{2}$ uptake by chemical weathering of crustal minerals is assumed to depend on surface temperature and the partial pressure of $\mathrm{CO}_{2}$ as follows;

$$
W=\iint W_{0}\left(\frac{\mathrm{pCO}_{2}}{\mathrm{pCO}_{2}{ }^{*}}\right) \exp \left\{\frac{-\Delta E}{R T}\right\} d \phi d t .
$$

Here, $W$ is the rate of the uptake of $\mathrm{CO}_{2}$ by chemical weathering, $\Delta E$ is activation energy of weathering, and $R$ is the gas constant (e.g., Walker et al. 1981).

\section{Results}

The climates of eccentric planets can be classified into seven climate modes: Runaway Greenhouse (RG), Ice-free (IF), Seasonally Ice-cap (SIC), Ice-cap (IC), Seasonally Snowball (SSB), Snowball (SB), and $\mathrm{CO}_{2}$ Cloud (CO) modes. The RG and CO modes cannot be studied with our models. They are only defined by the annual mean solar flux. The IF mode planets have no ice cap, and the IC mode planets are partially ice-covered. The SIC planets have no ice when they are around perihelion, but become partially ice-covered when they are around aphelion. The SSB planets are globally ice-covered when they are around aphelion. The planets under these four climate modes have liquid water on their surfaces, at least seasonally. On the other hand, the SB mode planets become globally ice-covered repeatedly and have no liquid water on their surfaces.

Figure 1 shows the climate mode as functions of semi-major axis and eccentricity when the $\mathrm{CO}_{2}$ degassing rate is equal to the present Earth's value. Dotted lines in Figure 1

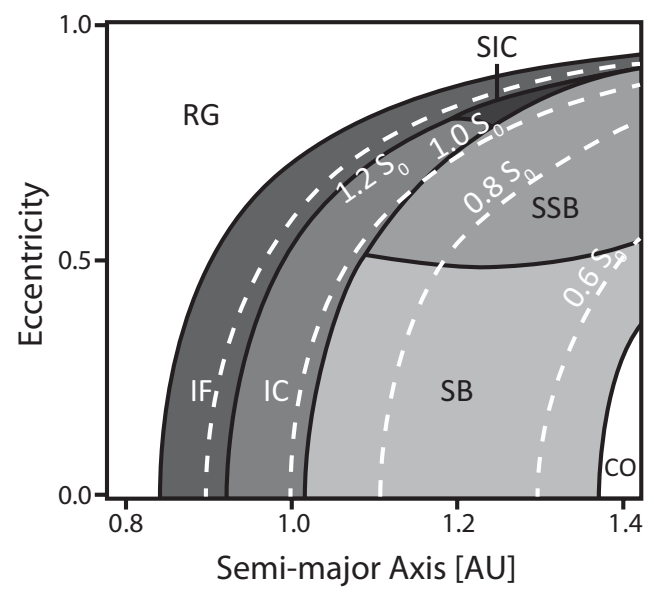

Figure 1. Climate mode as functions of semi-major axis and eccentricity. The $\mathrm{CO}_{2}$ degassing rate is equal to the present Earth's value. Dotted lines are contours of annual mean insolation. 


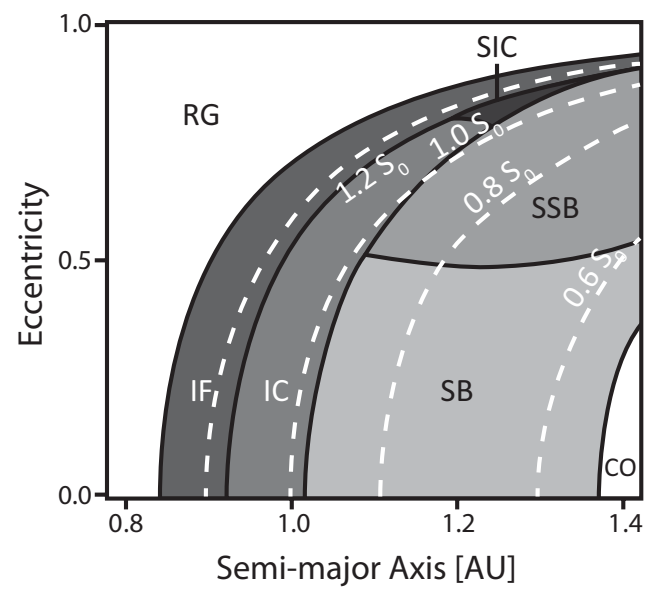

Figure 2. Climate mode as functions of semi-major axis and eccentricity. The $\mathrm{CO}_{2}$ degassing rate is 10 times the present Earth's rate.

are contoura representing annual mean insolation, which suggests that the boundaries among IF, IC and SB modes depend on the annual mean insolation as shown by Williams \& Pollard (2002) even when we consider carbonate-silicate geochemical cycle. This is because large heat capacity of oceans suppresses the influence of seasonal change of insolation. With the degassing rate of $\mathrm{CO}_{2}$ as much as the present value of Earth, the planets are under the SB mode when they are orbiting farther than 1.02 AU from a Sunlike star. Under such conditions, the planet has higher partial pressure of $\mathrm{CO}_{2}$, which makes $\mathrm{CO}_{2}$ uptake by silicate weathering larger. This results in the $\mathrm{CO}_{2}$ degassing flux not to be balanced by the $\mathrm{CO}_{2}$ uptake flux by silicate weathering, resulting in the planet to be in the SB mode. To avoid the SB mode, the $\mathrm{CO}_{2}$ degassing rate should be higher than the present Earth's rate (e.g., Figure 2).

Figure 2 shows the climate modes of the planets whose $\mathrm{CO}_{2}$ degassing rate is 10 times the present Earth's value. For this condition, climate modes also depend on the annual mean insolation. The difference between Figure 1 and 2 is that in Figure 2, the planets orbiting farther than 1.18 AU are also under the IF mode although the planets between 1.0 AU and 1.18 AU are under the IC mode. That is, the lower insolation the planets receive, the warmer the planets become. This paradoxical feature is derived from the high atmospheric $\mathrm{pCO}_{2}$ level due to carbonate-silicate geochemical cycle. Then $\mathrm{CO}_{2}$ atmosphere makes meridional heat transport more efficient, resulting in a shrink of icesheet and warmer climate.

\section{Conclusion}

We investigate the climate of the eccentric terrestrial planets with carbonate-silicate geochemical cycles. Our results suggest that the climates of the planets are dependent on the annual mean insolation, and that the planets with $\mathrm{CO}_{2}$ degassing as much as the present Earth's rate are globally ice-covered if they are orbiting farther than 1.02 AU. We found that, if the planets are far away from the central star with the $\mathrm{CO}_{2}$ degassing 10 times the present Earth's rate, they will be under the IF mode rather than the SB mode because of an efficient meridional heat transport of dense $\mathrm{CO}_{2}$ atmosphere. 


\section{References}

Dressing, C. D., Spiegel, D. S., Sharf, C. A., Menou, K., \& Raymond, S. N. 2010, ApJ, 721, 1295

North, G. R., Cahalan, R. F., \& Coakley Jr, J. A. 1981, Rev. Geophys. Space Phys., 19, 91

Walker, J. C. G., Hays, P. B., \& Kasting, J. F. 1981, J. Geophys. Res., 86, 9776

Williams, D. M. \& Kasting, J. F. 1997, Icarus, 129, 254

Williams, D. M. \& Pollard, D. 2002, Int. J. Astrobiol., 1, 61 